\title{
Transformation of sinusoidal electromagnetic and polarization waves into cnoidal waves in an optical fibre
}

\author{
Dzedolik I.V. \\ Taurida V. Vernadsky National University, 4 Vernadsky Ave., \\ 95007 Simferopol, Ukraine, e-mail: dzedolik@crimea.edu
}

Received: 04.08.2008

\begin{abstract}
It is shown that a sinusoidal signal wave can transform into a cnoidal (periodic) wave in an optical fibre if the power signal propagates in the fibre core. In this case the signal wave period increases, but the wave profile does not almost change. The signal wave gets the form of kink for the high-power signal. The wave transformation is determined by nonlinear effects in the fibre core. The linear modes of the fibre transform into the nonlinear ones under influence of cubic or higher nonlinear interactions of electromagnetic and polarization waves. These changes are described by the equation with sinusoidal nonlinearity for the polarization wave. The approximate and exact analytical solutions of the nonlinear equation are obtained.
\end{abstract}

PACS: 42.65.-k, 42.65.Wi

UDC: 535.3

Keywords: optical fibre, polariton, cnoidal wave, kink

\section{Introduction}

Nowadays optical fibres have become basic media for transmitting information streams. The more information you transfer the higher power of input signals you deal with. As a result, nonlinear interactions of electromagnetic waves and dielectric medium can take place in the core of optical fibre. The critical value of intensity needed for appearance of nonlinear polarization waves is $I \sim 10^{12} \mathrm{~W} / \mathrm{cm}^{2}$ [1, 2]. In addition, cumulative nonlinear effects appear at long distances in fibres even if the transmitted signals have relatively low powers. Since the electromagnetic and polarization waves do not always look like linear modes in fibres [3], nonlinear polariton patterns have been examined as nonlinear fibre modes [4-8].

There are several well-known methods for analysis of signal propagation in different dielectric waveguides and optical fibres: analytical [1, 2, 4, 6, 8], semi-analytical [2, 5, 8] and numerical $[2,7,8]$. In the present work we consider a new aspect of the analytical method for analysing linear and nonlinear polariton waves generated in cylindrical dielectric waveguides or optical fibres. 
We will show that a sinusoidal polariton wave can transform into a cnoidal (periodic) one whenever a high-power signal propagates in the fibre core. Then the period of the polariton wave increases, but the wave profile remains almost the same. If the signal power becomes very high, the polariton wave gets a form of kink, i.e. we deal with a socalled shock wave. The changes in the wave profile and the period are revealed at the output of the transmission line. All the wave transformation effects are determined by nonlinearity in the fibre core. These changes of the wave profile and the period could be described by equation with a sinusoidal nonlinearity for the polariton wave.

\section{Axial symmetric TE-modes}

Let us consider the conditions for appearance of nonlinear polarization waves in a quartz optical fibre. The polarization waves are generated in the fibre by falling electromagnetic waves, the waves of polarization generate the electromagnetic waves in the medium and so on. The Maxwell equations describe the processes of the wave generation as follows:

$$
\begin{aligned}
& \nabla \times \vec{B}=\frac{1}{c} \frac{\partial \vec{D}}{\partial t}, \quad \nabla \vec{D}=0, \\
& \nabla \times \vec{E}=-\frac{1}{c} \frac{\partial \vec{B}}{\partial t}, \quad \nabla \vec{B}=0,
\end{aligned}
$$

with the medium equation $\vec{D}=\vec{E}+4 \pi \vec{P}$, where $\vec{P}=-e N \vec{r}$ is the polarization vector and $N$ the number of atoms per unit volume. The equation for the electromagnetic and polarization waves may be obtained from Eqs. (1) and the medium equation:

$$
\left(\nabla^{2}-\frac{1}{c^{2}} \frac{\partial^{2}}{\partial t^{2}}\right) \vec{E}=-4 \pi\left[\nabla(\nabla \vec{P})-\frac{1}{c^{2}} \frac{\partial^{2} \vec{P}}{\partial t^{2}}\right] .
$$

The fused quartz $\mathrm{SiO}_{2}$ included in the optical fibre is amorphous material. The unit cell of quartz includes the inversion centre, so that the potential of its lattice near each atom can be approximated by the even periodic function $U=-f_{0} \cos \left(2 \pi r / b_{0}\right)$, where $b_{0}$ is the average atomic distance. We can write the motion equation for the external electron of $\mathrm{Si}$ or $\mathrm{O}$ ions in quartz as

$$
m \frac{d^{2} \vec{r}}{d t^{2}}=-\nabla U-e \vec{E}
$$

where $-\nabla U=-\vec{f}_{0} \quad \sin \left(2 \pi \quad r / b_{0}\right)$ is the average restoring force of the lattice. Let us suppose that the frequency of the periodic force lies far away from the dissipation region of quartz. Then we multiply Eq. (3) by ( $-e N)$ and divide by the electron mass $m$. We obtain the following equation for the polarization wave:

$$
\frac{d^{2} \vec{P}}{d t^{2}}=-\vec{f}_{m} \sin (\vec{q} \vec{P})+v_{e}^{2} \vec{E},
$$


where $\vec{f}_{m}=e N \vec{f}_{0} / m, \vec{q}=\overrightarrow{1}\left(2 \pi / b_{0} e N\right)$ and $v_{e}^{2}=e^{2} N / m$. Eqs. (2) and (4) describe nonlinear modes of an optical fibre $[4,8]$. The magnetic field can be obtained from the expression $\vec{B}=-c \nabla \times \int d t \vec{E}$ after solving the set of Eqs. (2) and (4).

Now we will find solution of the set of Eqs. (2) and (4) for the optical fibre with the step refraction index profile. Suppose that axially symmetric TE-modes [3] propagating along $z$ axis are excited, with the vectors $\vec{E}_{\varphi}(r, z, t), \vec{P}_{\varphi}(r, z, t), \vec{B}_{r}(r, z, t), \vec{B}_{z}(r, z, t)$ in the cylindrical frame of reference $(r, \varphi, z)$. Taking into account the expression $\nabla \vec{P}=0$, we obtain the set of equations for both the electromagnetic and polarization waves (hereafter we drop the index $\varphi$ of vectors $\left.E_{\varphi}, P_{\varphi}\right)$ :

$$
\begin{aligned}
& \nabla_{r}^{2} E+\left(\frac{\partial^{2}}{\partial z^{2}}-\frac{1}{c^{2}} \frac{\partial^{2}}{\partial t^{2}}\right) E=\frac{4 \pi}{c^{2}} \frac{\partial^{2} P}{\partial t^{2}}, \\
& \frac{\partial^{2} P}{\partial t^{2}}+f_{m} \sin (q P)=v_{e}^{2} E .
\end{aligned}
$$

\section{Polariton spectra}

Let us represent the electric and polarization fields as $E=F(r) \tilde{E}(t, z), P=F(r) \tilde{P}(t, z)$. Inserting these variables into the first of Eqs. (5) and separating the functions $F(r)$ and $\tilde{E}(t, z), \tilde{P}(t, z)$, one can obtain

$$
\begin{aligned}
& \nabla_{r}^{2} F+s^{2} F=0, \\
& \left(\frac{\partial^{2}}{\partial z^{2}}-\frac{1}{c^{2}} \frac{\partial^{2}}{\partial t^{2}}+s^{2}\right) \tilde{E}=\frac{4 \pi}{c^{2}} \frac{\partial^{2} \tilde{P}}{\partial t^{2}},
\end{aligned}
$$

where $s$ is a constant. The first of Eqs. (6) defines the dependence on transversal coordinates for the functions $E$ and $P$ in the fibre [3].

To the first approximation we can consider the harmonic waves $\tilde{E}=E_{0} \exp (i \phi), \tilde{P}=P_{0} \exp (i \phi)$, where $\phi=\omega t-\beta z$ is the phase, $E_{0}, P_{0}=$ const the wave amplitudes and the constant $s$ is included in the propagation constant $\beta \rightarrow \beta+s$. In this case we can arrive at the dispersion law, if we expand the sine in the second of Eqs. (5) as $\sin (q P) \approx q P, q P<<1$ (the linear case) and use the second of Eqs. (6):

$$
\left(\frac{\omega^{2}}{c^{2}}-\beta^{2}\right)\left(\omega^{2}-f_{m} q\right)=\frac{\omega^{2} \omega_{e}^{2}}{c^{2}},
$$

where $\omega_{e}^{2}=4 \pi e^{2} N / m$ is the electronic plasma frequency. The solutions of Eq. (7) are two polariton branches (see Fig. 1, solid curves 1 and 2): 


$$
\omega_{ \pm}=\left\{\frac{\left(\omega_{e}^{2}+f_{m} q+c^{2} \beta^{2}\right)}{2} \pm\left[\frac{\left(\omega_{e}^{2}+f_{m} q+c^{2} \beta^{2}\right)^{2}}{4}-c^{2} f_{m} q \beta^{2}\right]^{1 / 2}\right\}^{1 / 2} .
$$

To the second approximation the polariton branches acquire dependence on the amplitude of the polarization wave $P_{0}$, if we consider that $\sin (q P) \approx q P-\left(q^{3} / 6\right) P_{0}^{2} P$ (nonlinear case - see Fig. 1, dotted curves 1 and 2)

$$
\begin{aligned}
\omega_{ \pm} & =\left[\frac{\omega_{e}^{2}+f_{m}\left(q-q^{3} P_{0}^{2} / 6\right)+c^{2} \beta^{2}}{2} \pm\right. \\
& \left.\left\{\frac{\left(\omega_{e}^{2}+f_{m}\left(q-q^{3} P_{0}^{2} / 6\right)+c^{2} \beta^{2}\right)^{2}}{4}-c^{2} f_{m}\left(q-q^{3} P_{0}^{2} / 6\right) \beta^{2}\right\}^{1 / 2}\right]^{1 / 2}
\end{aligned}
$$

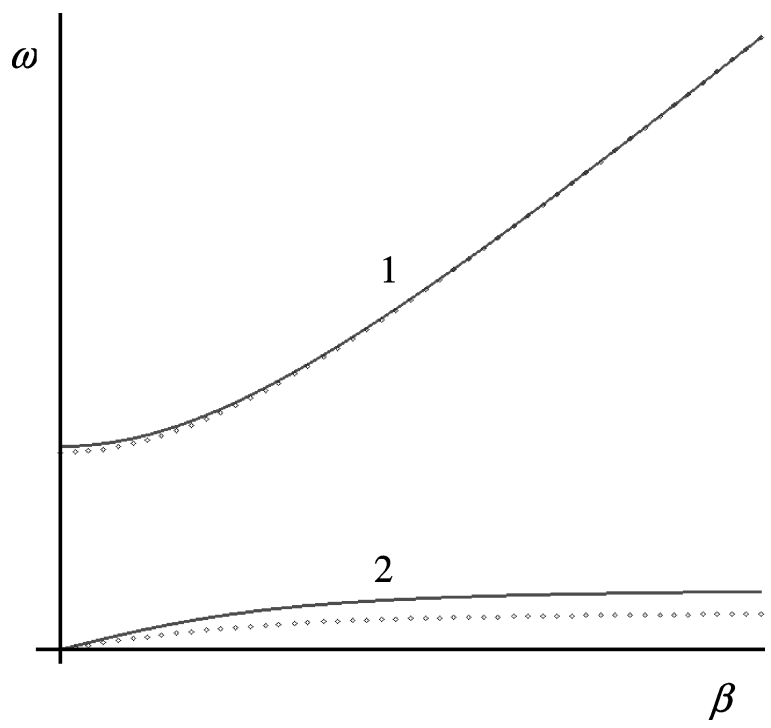

Fig. 1. Branches of polariton spectrum for an optical fibre: $1-\omega_{+}, 2-\omega_{-}$.

Solid and doted curves refer respectively to linear and nonlinear cases.

\section{Nonlinear polariton waves}

Let us rewrite the second of Eqs. (6) in the form

$$
\left(\frac{\partial^{2}}{\partial z^{2}}-\frac{1}{c^{2}} \frac{\partial^{2}}{\partial t^{2}}+s^{2}\right) E=\frac{4 \pi}{c^{2}} \frac{\partial^{2} P}{\partial t^{2}} .
$$

We can change the variables $t, z \rightarrow \phi$ and then obtain from Eqs. (5) and (10) the following set of equations: 


$$
\begin{aligned}
& \frac{d^{2} E}{d \phi^{2}}=\frac{4 \pi \omega^{2}}{c^{2} \beta^{2}-\omega^{2}} \frac{d^{2} P}{d \phi^{2}}, \\
& \frac{d^{2} P}{d \phi^{2}}+\frac{f_{m}}{\omega^{2}} \sin (q P)=\frac{v_{e}^{2}}{\omega^{2}} E .
\end{aligned}
$$

From the first of Eqs. (11) we get the integral of motion $I_{E P}$ in the $\phi$-space:

$$
E=\frac{4 \pi \omega^{2}}{c^{2} \beta^{2}-\omega^{2}} P+I_{E P}
$$

We can neglect the constant $I_{E P}$ in Eq. (12) for the relation of the electric field and polarization, if we assume that the polarization is excited by the alternating field only. Then one can insert Eq. (12) into the second of Eqs. (11) and obtain the equation for polarization:

$$
\frac{d^{2} P}{d \phi^{2}}+f_{S} \sin (q P)+a_{0} P=0
$$

where $f_{S}=f_{m} / \omega^{2}$ and $a_{0}=\omega_{e}^{2} /\left(\omega^{2}-c^{2} \beta^{2}\right)$.

The solution of Eq. (13) may be obtained with different methods. One of them is averaging over periodic variable such as the fast oscillating phase. For this purpose we can use the Van der Pole method [9]. We present the solution of equation in the form $P=P_{0}(\phi) e^{i \phi}$, multiply the equation by $e^{-i \phi}$ and average it over the period $2 \pi$ :

$$
\frac{d^{2} P_{0}}{d \phi^{2}}+i 2 \frac{d P_{0}}{d \phi}+\left(a_{0}-1\right) P_{0}+\frac{f_{S}}{2 \pi} \int_{0}^{2 \pi} d \phi e^{-i \phi} \sin \left(q P_{0} e^{i \phi}\right)=0 .
$$

If we expand the sine in Eq. (14) in the Taylor series up to the cubic term $\left(\sin (q P) \approx\left[q P_{0}-\left(q^{3} / 6\right) P_{0}^{3}\right] e^{i \phi}\right)$ and disregard the second derivative of $P_{0}$ in the spirit of the method mentioned, we obtain

$$
\phi=i \frac{12}{f_{S} q^{3}} \int_{P(0)}^{P_{0}} \frac{d x}{x\left(x^{2}+c_{0}^{2}\right)}=\frac{i}{1-a_{0}-f_{S} q} \ln \frac{\mathrm{x}^{2}}{\mathrm{x}^{2}+c_{0}^{2}} \mid \begin{gathered}
P_{0} \\
P(0)
\end{gathered},
$$

where $c_{0}^{2}=6\left(1-a_{0}-f_{S} q\right) / f_{S} q^{3}$. The approximate solution of Eq. (14) for the polarization wave amplitude follows from the expression obtained above:

$$
P_{0}=\frac{c_{0} \exp (i \tilde{\phi} / 2)}{\sqrt{d_{0}^{2}-e^{i \tilde{\phi}}}}
$$


where $d_{0}^{2}=\left[c_{0}^{2}+P^{2}(0)\right] / P^{2}(0)=$ const and $\tilde{\phi}=\left(a_{0}+f_{S} q-1\right) \phi$. In this approximation, the polarization wave looks as

$$
P=\frac{c_{0} F(r)}{\sqrt{d_{0}^{2}-\exp \left[i\left(a_{0}+f_{S} q-1\right) \phi\right]}} \exp \left[i\left(a_{0}+f_{S} q+1\right) \frac{\phi}{2}\right] .
$$

We can rewrite this expression in the form

$$
\begin{aligned}
& P=\frac{c_{0} F(r)}{\sqrt{1+d_{0}^{4}-2 d_{0}^{2} \cos \left[\left(a_{0}+f_{s} q-1\right) \phi\right]}} \times \\
& \exp \left\{i\left[\left(a_{0}+f_{S} q+1\right) \frac{\phi}{2}-\frac{\sin \left[\left(a_{0}+f_{s} q-1\right) \phi\right]}{d_{0}^{2}-\cos \left[\left(a_{0}+f_{S} q-1\right) \phi\right]}\right]\right\},
\end{aligned}
$$

and so obtain the electric field from Eqs. (12) and (15):

$$
\begin{aligned}
& E_{\varphi}=\frac{4 \pi \omega^{2} c_{0} F(r)}{\left(c^{2} \beta^{2}-\omega^{2}\right) \sqrt{1+d_{0}^{4}-2 d_{0}^{2} \cos \left[\left(a_{0}+f_{S} q-1\right) \phi\right]}} \times \\
& \exp \left\{i\left[\left(a_{0}+f_{S} q+1\right) \frac{\phi}{2}-\frac{\sin \left[\left(a_{0}+f_{S} q-1\right) \phi\right]}{d_{0}^{2}-\cos \left[\left(a_{0}+f_{S} q-1\right) \phi\right]}\right]\right\} .
\end{aligned}
$$

\section{Exact solutions for the cnoidal polariton waves}

Another method for solving Eq. (13) is its direct integration with the initial conditions $P(\phi=0)=0,(d P / d \phi)_{0}=\xi=$ const . The first integral of Eq. (13) is

$$
\left(\frac{d P}{d \phi}\right)^{2}=\xi^{2}+\frac{2 f_{S}}{q}[\cos (q P)-1]-a_{0} P^{2} .
$$

If the electric field is small, the polarization of medium will be small, too $(q P<<1)$. Then we expand the cosine in Eq. (17) into a Taylor series $\left(\cos (q P) \approx 1-\frac{q^{2} P^{2}}{2}\right)$ and obtain the second integral for the linear case:

$$
\phi=\int \frac{d P}{\sqrt{\xi^{2}-\left(a_{0}+f_{S} q\right) P^{2}}}=\left\{\begin{array}{l}
\arcsin \left(P / b_{0}\right), \\
\arccos \left(P / b_{0}\right),
\end{array}\right.
$$

where $b_{0}=\frac{\xi}{\sqrt{a_{0}+f_{S} q}}$. After converting the function in Eq. (18) we get trigonometric functions describing the polarization wave: 


$$
P= \begin{cases}b_{0} \sin \left(\sqrt{a_{0}+f_{S} q}\right. & \phi), \\ b_{0} \cos \left(\sqrt{a_{0}+f_{S} q}\right. & \phi) .\end{cases}
$$

It is clear that the polarization wave is described as a harmonic function in the linear case, i.e. the optical fibre modes are then linear. The solutions of Eqs. (19) may be considered as a test of the method suggested.

Let us now consider the next term in the series of cosine $\left(\cos (q P) \approx 1-q^{2} p^{2} / 2+q^{4} P^{4} / 24\right)$. Then we obtain the following integral of Eq. (17):

$$
\phi=\int \frac{d P}{\sqrt{\xi^{2}-\left(a_{0}+f_{S} q\right) P^{2}+\left(f_{S} q^{3} / 12\right) P^{4}}} .
$$

This is the integral $\int_{0}^{x} \frac{d x}{\sqrt{\left(a^{2}-x^{2}\right)\left(b^{2}-x^{2}\right)}}=\frac{1}{a} \tilde{F}\left(\tilde{\varphi}, \frac{b}{a}\right)$, where notation $\tilde{F}(\tilde{\varphi}, b / a)$ means the elliptic integral of the first kind, $\tilde{\varphi}=\arcsin (x / b)$ and the constants $a$ and $b$ are defined from the equations $a^{2} b^{2}=\frac{12 \xi^{2}}{f_{S} q^{3}}, \quad a^{2}+b^{2}=\frac{12\left(a_{0}+f_{S} q\right)}{f_{S} q^{3}}$ :

$$
\begin{gathered}
a^{2}=\frac{\xi^{2}}{\left(a_{0}+f_{S} q\right) / 2 \pm \sqrt{\left(a_{0}+f_{S} q\right)^{2} / 4-\xi^{2}}}, \\
b^{2}=\frac{6\left(a_{0}+f_{S} q\right)}{f_{S} q^{3}} \pm \sqrt{\frac{36\left(a_{0}+f_{S} q\right)^{2}}{f_{S}^{2} q^{6}}-\frac{12 \xi^{2}}{f_{S} q^{3}}}
\end{gathered}
$$

We convert the elliptic integral to the Jacobi sine, sn, and obtain the expression for the nonlinear polarization wave in the form of cnoidal periodic wave:

$$
P=b \cdot \operatorname{sn}\left(a \sqrt{\frac{f_{s} q^{3}}{12}} \phi, \frac{b}{a}\right) .
$$

The cnoidal wave transforms to the sinusoidal one (i.e., the linear mode of optical fibres) if only $\frac{b}{a} \rightarrow 0$. Then we have the solution $\operatorname{sn}\left(a \sqrt{\frac{f_{S} q^{3}}{12}} \phi, \frac{b}{a}\right) \rightarrow \sin \left(a \sqrt{\frac{f_{S} q^{3}}{12}} \phi\right)$, with a larger period (see Fig. 2). This means that the period of signal wave depends on its power and the wave period increases when the signal intensity in the fibre does. Otherwise, the signal wave period can change if the initial condition $\xi=(d P / d \phi)_{0}$ is varied at 
the fibre input. For example, if the module of $s n$ approaches unity $\left(\frac{b}{a} \rightarrow 1\right)$, then the following wave transformation takes place: $s n\left(a \sqrt{\frac{f_{S} q^{3}}{12}} \phi, \frac{b}{a}\right) \rightarrow \operatorname{tn}\left(a \sqrt{\frac{f_{S} q^{3}}{12}} \phi, 1\right)$, i.e. a shock wave (kink) arises from the cnoidal wave (see Fig. 3).

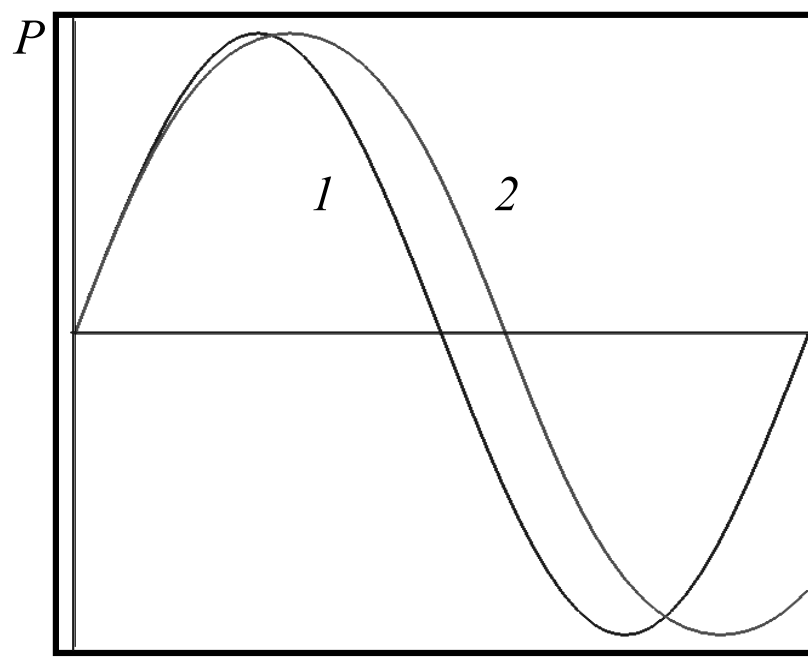

Fig. 2. Polarization waves: curves 1 and 2 correspond respectively to sinusoidal and cnoidal waves.

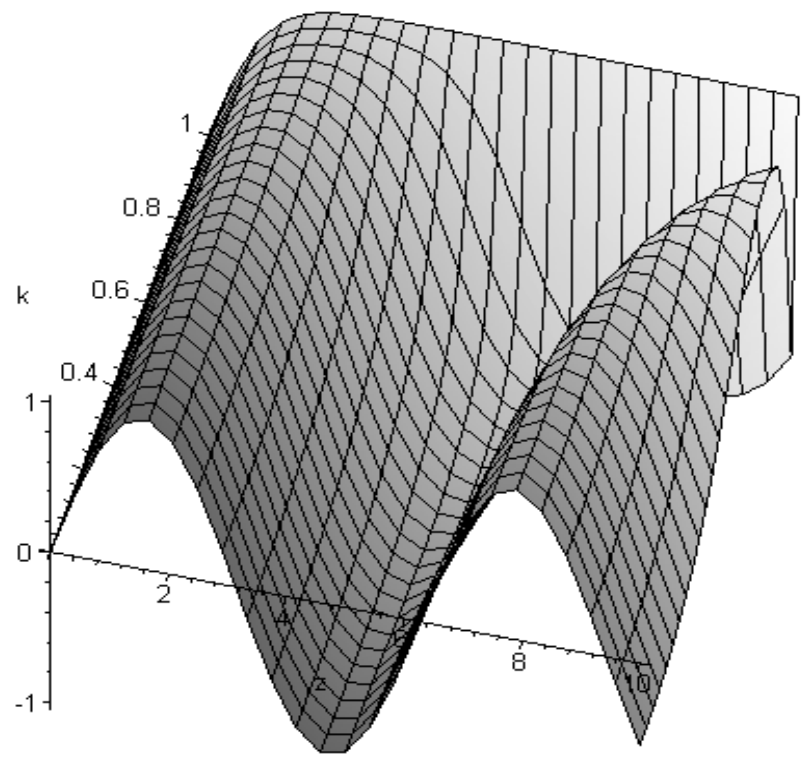

Fig. 3. Transformation of cnoidal wave into a kink by varying the module $k=b / a$.

Now we find the electric field from Eqs. (12) and (21):

$$
E_{\varphi}=\frac{4 \pi \omega^{2} b}{c^{2} \beta^{2}-\omega^{2}} F(r) \operatorname{sn}\left(a \sqrt{f_{S} q^{3} / 12} \phi, \frac{b}{a}\right) .
$$


One can integrate Eq. (17) without expanding the cosine
$\left(\phi=\int_{0}^{P} \frac{d x}{\sqrt{g-a_{0} P^{2}+\left(2 f_{S} / q\right) \cos (q P)}}\right.$, where $\left.g=\xi^{2}-2 f_{S} / q\right)$ and obtain the following expression for the case $a_{0} \rightarrow 0$ :

$$
\phi=-\frac{2}{4 f_{S}-q \xi^{2}} \tilde{F}\left[\cos \left(\frac{q P}{2}\right), \sqrt{\frac{4 f_{S}}{4 f_{S}-q \xi^{2}}}\right] .
$$

After converting Eq. (23) one can get the expressions for the cnoidal polariton wave described with the Jacobi cosine:

$$
P=\frac{2}{q} \operatorname{cn}\left(\frac{4 f_{S}-q \xi^{2}}{2} \phi, \sqrt{\frac{4 f_{S}}{4 f_{S}-q \xi^{2}}}\right) .
$$

A transformation of the cnoidal wave given by Eq. (24) to a soliton takes place $\left(c n\left[\frac{\left(4 f_{S}-q \xi^{2}\right) \phi}{2}, \tilde{k}\right] \rightarrow \sec h\left(\frac{\left(4 f_{S}-q \xi^{2}\right) \phi}{2}\right)\right.$ ) if the module $\tilde{k} \rightarrow 1$ tends to unity. Finally, one can find the electric field from Eq. (12) and Eq. (24) for the case of highpower signals:

$$
E_{\varphi}=\frac{8 \pi \omega^{2}}{q\left(c^{2} \beta^{2}-\omega^{2}\right)} F(r) c n\left(\frac{4 f_{S}-q \xi^{2}}{2} \phi, \sqrt{\frac{4 f_{S}}{4 f_{S}-q \xi^{2}}}\right) .
$$

\section{Conclusions}

Electromagnetic wave in an optical fibre can acquire a form of polariton wave. By injecting a powerful signal into the fibre we get transformation of the harmonic signal wave into the cnoidal, kink or soliton ones. The period of high-power polariton wave increases and the wave profile changes, depending on the parameters of both the wave and the fibre.

All the wave transformation effects are determined by nonlinear properties of the fibre core. The nonlinear changes in the wave period and profile are described by the equations for polariton waves with sinusoidal nonlinearity. These equations allow for finding exact solutions.

The polariton spectrum has the two branches each of which is related to the linear and nonlinear cases. The branches referred conventionally to as "plus" are similar for the both cases; though the "minus" branches are different (the nonlinear branch is lower than the linear one).

Hence, the analysis of signal propagation developed by us, which is based on the equation with sinusoidal nonlinearity for the polariton wave, provides more clear under- 
standing of signal disturbances in the transmitting lines and optical sensors made from the optical fibres. While operating with several input signals, one should take into account a possibility of reading data errors because the overall power of all the signals can influence their periods. In order to avoid these errors while receiving the data, one should analyze the period of signal as a function of its power.

\section{Acknowledgment}

The author thanks V A Kochelap and the researchers of Theoretical Department of Institute of Semiconductor Physics (Kiev, Ukraine) for their fruitful comments on this work.

\section{References}

1. Sukhorukov A P. Nonlinear wave interactions in optics and radio physics. Moscow: Nauka (1988).

2. Kivshar Yu S and Agrawal G P. Optical Solitons: From Fibres to Photonic Crystals. Academic Press (2003).

3. Snyder A W and Love J D. Optical waveguide theory. London, New York: Chapman and Hall (1983).

4. Dzedolik I V and Dzedolik A I, 2002. Nonlinear modes in optical fibres. Technical Physics Letters. 28(5): 403-405.

5. Zheltikov A, 2005. Gaussian-mode analysis of waveguide-enhanced Kerr-type nonlinearity of optical fibres and photonic wires. J. Opt. Soc. Am. B. 22(5): $1100-1104$.

6. Qi X, Zhang X, Wei H and Plant D V, 2006. Linearity of nonlinear perturbations in fibre-optic transmission lines and its applications to nonlinear compensations. J. Opt. Soc. Am. B. 23(10): 2032-2039.

7. Korneev $\mathrm{N}$ and Marroquin F, 2007. Long-distance propagation of periodic patterns in weakly nonlinear Kerr medium. J. Opt. Soc. Am. B. 24(1): 84-89.

8. Dzedolik I V. Polaritons in optical fibres and dielectric resonators. Simferopol: DIP (2007).

9. Nayfeh A H. Perturbation methods. New York-London-Sydney-Toronto: John Wiley and Sons (1973). 